\title{
Zeno and anti-Zeno effects in multimode parametric down-conversion
}

\author{
Alfredo Luis* \\ Departamento de Óptica, Facultad de Ciencias Físicas, Universidad Complutense, 28040 Madrid, Spain \\ (Received 14 October 2001; published 2 July 2002)
}

\begin{abstract}
We present a simple multimode analysis of the Zeno and anti-Zeno effects in spontaneous parametric down-conversion. In this process the input vacuum is an unstable state decaying into a reservoir of downconverted modes by emitting a pair of photons. We show that the Zeno and the anti-Zeno effects are two particular examples of quantum propagation depending on the effective spectrum of down-converted modes. We show that this evolution can be easily tailored on demand and that these quantum phenomena admit a very simple explanation in terms of standard interference concepts.
\end{abstract}

DOI: 10.1103/PhysRevA.66.012101

PACS number(s): 03.65.Xp, 42.50.-p

\section{INTRODUCTION}

Measurement is a very fundamental issue of quantum theory that supplies distinct examples of purely quantum behavior without classical analog. For example, one of the appealing consequences of quantum mechanics is that the observation unavoidably disturbs the observed system. This is particularly revealed by the so-called Zeno and anti-Zeno effects [1-6]. The Zeno (anti-Zeno) effect refers to the inhibition (acceleration) of the evolution of a dynamical system when attempts are made to observe it.

The most paradigmatic example considers the alteration of the spontaneous and irreversible decay of an unstable state, such as the radiative decay of an excited atom or the radioactive decay of a nucleus. However, the experimental observation of truly decaying systems encounters difficulties so that most efforts have focused on reversible processes such as the Rabi oscillation in two-level atoms [7]. Nevertheless, the observation of the Zeno effect for unstable systems has been reported recently [8].

In this work we analyze a simple physical process where spontaneous decay can easily be observed and studied in very different quantum regimes. This is the generation of pairs of photons in parametric down-conversion in a nonlinear crystal [9]. This process has been used in a large number of fundamental experiments in quantum optics [10]. We present a multimode analysis of the Zeno and anti-Zeno effects in spontaneous parametric down-conversion. In this process the input vacuum can be regarded as an unstable state that decays into a reservoir of down-converted modes by emitting a pair of photons. This analogy allows us to carry out an analysis of parametric down-conversion that parallels general approaches to the decay of unstable systems such as the one developed in Ref. [5].

The general multimode approach carried out here demonstrates that the Zeno and anti-Zeno effects are particular cases of a general alteration of the photon emission caused by observation. Previous monomode approaches $[3,11]$ are recovered as particular limits. We show that this multimode analysis provides simple formulas to suitably tailor the reservoir spectrum, which in turn controls the decay. This con-

*Electronic address: alluis@ fis.ucm.es firms that parametric down-conversion provides a simple and accessible framework to study theoretically and experimentally the Zeno and anti-Zeno effects for a truly decaying system. In particular, we show that these quantum phenomena admit a very simple explanation in terms of standard interference concepts.

In Sec. II we obtain useful expressions for the unobserved emission rate of down-converted photons. In Sec. III we show how a simple observation scheme modifies the emission rate, leading to the Zeno and anti-Zeno effects depending on the observation regime. Finally, in Sec. IV we derive suitable monomode limits.

\section{UNOBSERVED DECAY RATE}

We consider a nonlinear crystal pumped by a strong, classical, and coherent field to produce pairs of photons via spontaneous down-conversion. Throughout we assume the parametric approximation in which the pump mode is strong enough so that it can be described classically by a constant complex amplitude. On the other hand, the down-converted modes are initially in the vacuum state $|0\rangle$.

The dynamics of this system can be described conveniently by using the effective momentum operator [12]

$$
\begin{aligned}
G= & -\hbar \sum_{k^{\prime}, k^{\prime \prime}}\left[\chi\left(\omega^{\prime}, \omega^{\prime \prime}\right) e^{i \Delta k z} a_{k^{\prime}}^{\dagger} a_{k^{\prime \prime}}^{\dagger}\right. \\
& \left.+\chi^{*}\left(\omega^{\prime}, \omega^{\prime \prime}\right) e^{-i \Delta k z} a_{k^{\prime}} a_{k^{\prime \prime}}\right]
\end{aligned}
$$

where $\chi$ is proportional to the nonlinear susceptibility of the crystal and to the complex amplitude of the pump mode, $\omega^{\prime}$ and $\omega^{\prime \prime}$ are the frequencies associated with the wave vectors $k^{\prime}$ and $k^{\prime \prime}$, respectively, $\Delta k=k_{p}-k^{\prime}-k^{\prime \prime}$ is the phase mismatch in the $z$ direction (for simplicity we assume perfect phase matching in the $x$ and $y$ directions) where $k_{p}$ is the wave number of the pump mode, and $a_{k^{\prime}}, a_{k^{\prime \prime}}$ denote the complex amplitude operators associated with the downconverted modes. We will assume the frequency resonance condition $\omega_{p}=\omega^{\prime}+\omega^{\prime \prime}$, where $\omega_{p}$ is the frequency of the pump mode. In this approach the state vector $|\psi(z)\rangle$ describing the state of the down-converted modes obeys the propagation equation 


$$
i \hbar \frac{\partial}{\partial z}|\psi\rangle=-G|\psi\rangle,
$$

where we have taken the $z$ axis as the direction of propagation. This is the quantum counterpart of the classical propagation equations [13].

Due to the mode coupling (1) the initial state $|0\rangle$ (which otherwise would be stable) is coupled to a reservoir so that it becomes unstable and decays spontaneously, emitting a pair of photons. Therefore, we can define the survival probability as $P(z)=|\langle 0 \mid \psi(z)\rangle|^{2}$. Our objectives are to obtain $P(z)$ and to study how the observation modifies it.

To this end let us express $|\psi(z)\rangle$ as

$$
|\psi(z)\rangle=\alpha(z)|0\rangle+\sum_{k^{\prime}, k^{\prime \prime}} \beta_{k^{\prime}, k^{\prime \prime}}(z)\left|k^{\prime}, k^{\prime \prime}\right\rangle+\cdots,
$$

where $\left|k^{\prime}, k^{\prime \prime}\right\rangle$ denote states with a photon in mode $k^{\prime}$ and another one in mode $k^{\prime \prime}$. The initial conditions at $z=0$ are $\alpha(0)=1$ and $\beta_{k^{\prime}, k^{\prime \prime}}(0)=0$. In most practical situations the nonlinear interaction is weak enough so that the generation of more than a photon pair has a negligible probability. In such a case the propagation equation (2) gives the following equation for $\alpha$ :

$$
\frac{d \alpha}{d z}=-\int_{0}^{z} d z^{\prime} e^{i k_{p}\left(z^{\prime}-z\right)} \Phi\left(z-z^{\prime}\right) \alpha\left(z^{\prime}\right),
$$

where

$$
\Phi(z)=\sum_{k^{\prime}, k^{\prime \prime}} e^{i\left(k^{\prime}+k^{\prime \prime}\right) z}\left|\chi\left(\omega^{\prime}, \omega^{\prime \prime}\right)\right|^{2}
$$

Since the emission rate is small, we can solve Eq. (4) iteratively, retaining only the terms up to $|\chi|^{2}$,

$$
\frac{d \alpha}{d z} \simeq-\int_{0}^{z} d z^{\prime} e^{-i k_{p} z^{\prime}} \Phi\left(z^{\prime}\right),
$$

so that

$$
\alpha(z) \simeq 1-\int_{0}^{z} d z^{\prime}\left(z-z^{\prime}\right) e^{-i k_{p} z^{\prime}} \Phi\left(z^{\prime}\right) .
$$

The survival probability is

$$
P(L)=|\alpha(L)|^{2} \simeq 1-R(L) L \simeq e^{-R(L) L},
$$

where $L$ is the effective length of the crystal and

$$
R(L)=\frac{1}{L} \int_{-L}^{L} d z^{\prime}\left(L-\left|z^{\prime}\right|\right) e^{-i k_{p} z^{\prime}} \Phi\left(z^{\prime}\right)
$$

is the rate of down-conversion processes expressed as the probability of decay per unit length. This decay rate can be expressed also as

$$
R(L)=\int_{-\infty}^{\infty} d k F(k, L) H(k)
$$

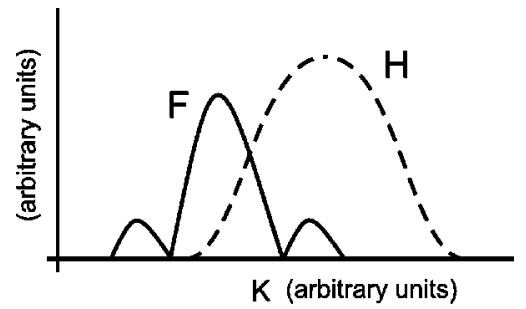

FIG. 1. Illustration of the overlap of the functions $F$ (solid) and $H$ (dashed) determining the decay rate. In this case the broadening of $F$ caused by the observation increases the overlap and leads to a larger decay rate (anti-Zeno effect).

where

$$
F(k, L)=L\left\{\frac{\sin \left[\left(k-k_{p}\right) L / 2\right]}{\left(k-k_{p}\right) L / 2}\right\}^{2}
$$

and

$$
H(k)=\sum_{k^{\prime}, k^{\prime \prime}}\left|\chi\left(\omega^{\prime}, \omega^{\prime \prime}\right)\right|^{2} \delta\left(k-k^{\prime}-k^{\prime \prime}\right) .
$$

This form (10) for the decay rate agrees with the results of Refs. [4,5]. This formal equivalence allows us to develop an analysis of the alteration of the photon emission in parametric down-conversion very similar to the one carried out in Ref. [5] for general unstable systems. This is accomplished in the next section.

\section{OBSERVED DECAY RATE}

Next we examine the survival probability when the moment of emission is observed. For the sake of simplicity we focus on the simplest observation procedure. Instead of a crystal of length $L$ we consider a cascade of $N$ crystals of length $L / N$ in series so that the total length of the nonlinear medium is always $L$. This splitting allows us to observe whether the emission has occurred or not after each piece. A possible implementation of this idea using a single crystal was proposed in Ref. [14]. The observation completely interrupts the down-converted modes so that the field state at the input of all the $N$ pieces is always the vacuum. The observed survival probability becomes

$$
\widetilde{P}_{N}(L)=[P(L / N)]^{N} \simeq e^{-R(L / N) L},
$$

and the observed decay rate is $\widetilde{R}_{N}(L)=R(L / N)$ while the unobserved one is $R(L)$.

In Eq. (10) it can be appreciated that the decay rate results from the overlap of the two functions $F$ and $H$, as illustrated in Fig. 1. The function $F$ is a phase-matching factor that contains the effect of the observation through its dependence on $L$. It is centered at the pump wave number $k_{p}$, the height of the central peak is $L / N$, and its width is proportional to $N / L$. Thus its width increases when the accuracy of the observation increases while the total area under $F$ is constant.

The function $H$ can be regarded as the reservoir spectrum of modes actually coupled to the unstable state. In many 
practical situations it can be assumed that $H$ has a Gaussian form and that $\chi\left(\omega^{\prime}, \omega^{\prime \prime}\right)$ has a maximum for $\omega^{\prime}=\omega^{\prime \prime}$ $=\omega_{p} / 2$. In such a case $H$ is centered at $\bar{k}=2 k\left(\omega_{p} / 2\right)$, i.e., twice the wave number corresponding to half the pump frequency (for simplicity we have assumed that the two downconverted waves experience the same index of refraction). In general $\bar{k} \neq k_{p}$ so that the maxima of $F$ and $H$ do not coincide,

$$
\bar{k}-k_{p}=2 k\left(\omega_{p} / 2\right)-k\left(\omega_{p}\right)=\frac{\omega_{p}}{c}\left[n_{c}\left(\omega_{p} / 2\right)-n_{p}\left(\omega_{p}\right)\right],
$$

where $n_{c}, n_{p}$ are the indices of refraction of the downconverted and pump modes, respectively.

Next we discuss how the survival probability of the initial vacuum state can be easily controlled by means of a suitable selection of the output modes leaving the crystals, which can be performed by means of frequency filters and diaphragms. The output modes that the filters interrupt are forced to be in the vacuum state when they reach the detector. If we interpret Eq. (3) as the output field state that reaches the detector we have that $\beta_{k^{\prime}, k^{\prime \prime}}=0$ for the modes interrupted by the filters. This effect can be formally taken into account in Eqs. (4) and (5) by multiplying $\chi$ by the transfer function describing the bandpass of the filters. This in turn leads to the replacement of $H$ in Eq. (10) by an effective reservoir function $H_{\text {eff }}$.

From Eq. (10) we have also the possibility of embodying the effect of the filters in the factor $F$ rather than in $H$. This possibility may seem even more natural since the filters are part of the detection arrangement. On the other hand, the idea of an effective mode reservoir $H_{\text {eff }}$ that can be easily tailored on demand can be fruitful because of the analogy between parametric down-conversion and the decay of general unstable systems developed above. For example, this allows us to regard the mode selection as a very simple and accessible practical implementation of the idea of reservoir engineering $[6,15]$. We can note also that the external mode selection would allow us to control very easily the effective mismatch depending on whether $k_{p}$ coincides or not with $2 k\left(\omega_{\max }\right)$, where $\omega_{\max }$ is the frequency of maximum transmission allowed by the filtering.

The main conclusion from these results is that the decay rate, and thus the existence of the Zeno or anti-Zeno effect, depends on the relative widths and centers of the functions $F$ and $H$, which in turn depend on the accuracy of the observation, and on the effective reservoir of down-converted modes.

More specifically, if the maxima of $F$ and $H$ differ significantly, as illustrated in Fig. 1, the broadening of $F$ caused by measurement increases the overlap between $F$ and $H$. Therefore $\widetilde{R}_{N}$ increases and $\widetilde{P}_{N}$ decreases (anti-Zeno effect). On the other hand, if their maxima are close enough and the width of $F$ is larger than that of $H$, as illustrated in Fig. 2, the effect of the observation is to decrease the height of $F$. Therefore the overlap decreases, $\widetilde{R}_{N}$ decreases, and $\widetilde{P}_{N}$ increases (Zeno effect). Finally, when their maxima are close

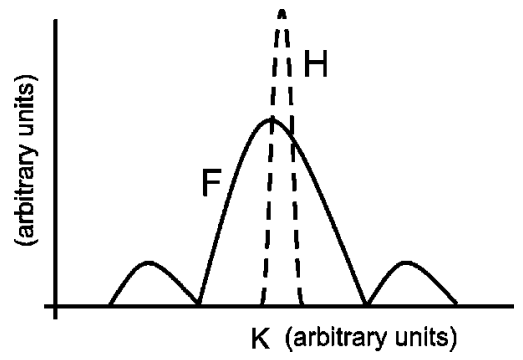

FIG. 2. Illustration of the overlap of the functions $F$ (solid) and $H$ (dashed) determining the decay rate. In this case the observation decreases the height of $F$, leading to a smaller overlap and a larger survival probability (Zeno effect).

enough and the width of $F$ is smaller than that of $H$, as illustrated in Fig. 3, the measurement has no effect. This is because the area under $F$ does not depend on $N$.

From a classical standpoint the strategy to infer the moment of emission that we have followed should not influence it. If after any piece the photons have not been emitted the field state is still the vacuum, while if the photons have been emitted the decay process has already finished before the detection takes place. This reasoning is based on a direct combination of particle behavior and randomness. However, the quantum behavior combines randomness with both particle and wave aspects and the probability of emission is the result of the interference of probability amplitudes [3,11]. Next we show that the quantum behavior analyzed in this work admits a very simple explanation in terms of basic and simple interference ideas.

The emission in the unobserved case results from the superposition of probability amplitudes originating in each part of the crystal. In principle they can interfere as long as they can be regarded as being emissions stimulated by the same input vacuum that imparts phase correlations between them. The actual coherence length of these probability amplitudes depends on the width of the mode spectrum $H$. When the centers of $F$ and $H$ coincide (phase matching) the interference is constructive. After interrupting the emission $N$ times, the probability amplitudes from each piece become mutually incoherent since they are stimulated by different vacuum modes. If the coherence length of the down-converted field is larger than $L / N$ (i.e., when $H$ is narrower than $F$ as illustrated in Fig. 2) this lack of coherence induced by measure-

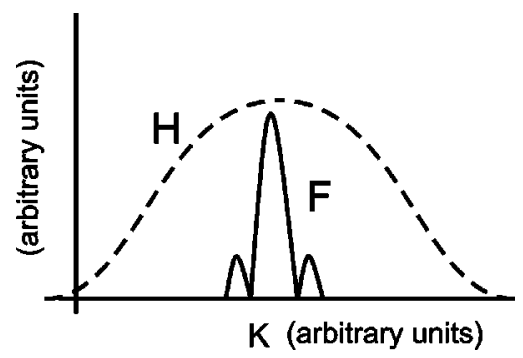

FIG. 3. Illustration of the overlap of the functions $F$ (solid) and $H$ (dashed) determining the decay rate. In this case the overlap does not depend appreciably on the accuracy of the observation since the area under $F$ is constant. 


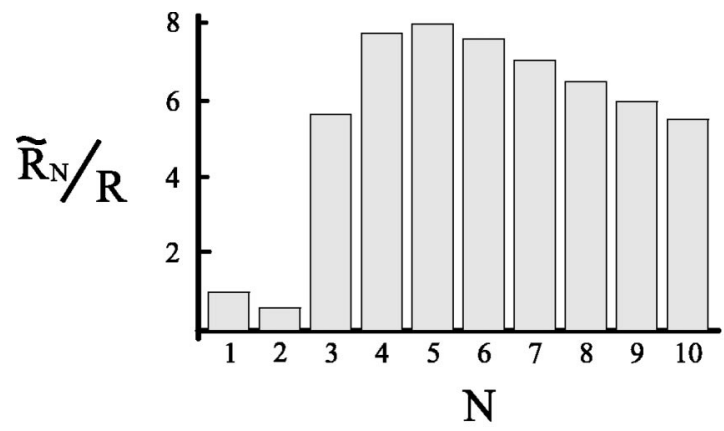

FIG. 4. Quotient between the observed $\widetilde{R}_{N}$ and unobserved $R$ decay rates as a function of the number $N$ of crystal pieces for $\left(k_{0}-k_{p}\right) L=11$. It can be seen that the maximum decay rate occurs for $N=5$ and hence the observed emission can occur faster than the unobserved one.

ment prevents constructive interference, the emission rate decreases and the survival probability increases (Zeno effect). Otherwise, when the coherence length of the downconverted field is shorter than $L / N$ (i.e., when $H$ is wider than $F$ as illustrated in Fig. 3) the emissions from different pieces of the crystal do not interfere from the very beginning and hence the measurement has no effect on the decay rate.

On the other hand, when the maxima of $F$ and $H$ do not coincide (phase mismatch) the interference is destructive. The loss of coherence induced by measurement can prevent this destructive interference and the probability of emission may increase as illustrated in Fig. 4 (anti-Zeno effect).

\section{MONOMODE LIMITS}

In this section we show that this general multimode analysis embraces previous monomode approaches presented in Refs. [3,11]. A monomode configuration can be obtained in the limit in which $H$ becomes a $\delta$ function $H(k)=\kappa \delta(k$ $-k_{0}$ ) for a given constant $k_{0}$, so that

$$
\widetilde{R}_{N}(L)=\frac{\kappa L}{N}\left\{\frac{\sin \left[\left(k_{0}-k_{p}\right) L /(2 N)\right]}{\left(k_{0}-k_{p}\right) L /(2 N)}\right\}^{2} .
$$

If $k_{0}=k_{p}$ (perfect phase matching)

$$
\widetilde{R}_{N}(L)=\frac{1}{N} R(L)
$$

and the observed survival probability $\widetilde{P}_{N}$ increases when $N$ increases, that is, when the accuracy of the monitoring of the emission increases. In the limit $N \rightarrow \infty$ the field state is always the vacuum irrespective of the total length $L$ of the nonlinear medium. This is the Zeno effect.

On the other hand, if $k_{0} \neq k_{p}$ (phase mismatch) the dependence on $N$ of the decaying rate $\widetilde{R}_{N}$ in Eq. (15) results from the competition of two effects. There is a direct dependence on $1 / N$ that tends to decrease the emission irrespective of the phase mismatch as in Eq. (16). There is another $N$ dependence in the argument of the sinc function that tends to increase the emission when $N$ increases. The net result is that there are $N$ values for which the observed emission rate is larger than the unobserved one as shown in Fig. 4 [3]. This is the anti-Zeno effect.

\section{CONCLUSIONS}

We have shown that parametric down-conversion provides a simple and experimentally accessible framework to study both the Zeno and anti-Zeno effects in a truly decaying system. One of the advantages of this arrangement is that it allows a very simple way of tailoring and controlling the variables that determine the decay. Furthermore, this practical scheme provides a very simple explanation of these quantum effects in terms of classical concepts such as interference and phase mismatch. Despite this simple picture, it must be stressed that parametric down-conversion is a completely quantum process having no classical analog. Classically, no down-converted fields are generated from the vacuum so that the decay of the initial state and its alteration caused by observation are genuine quantum processes.

\section{ACKNOWLEDGMENTS}

I thank Professor J. Peřina and Dr. J. Řeháček for a careful reading of the manuscript and valuable suggestions.
[1] D. Home and M.A.B. Whitaker, Ann. Phys. (N.Y.) 258, 237 (1997).

[2] A.M. Lane, Phys. Lett. 99A, 359 (1983); S.A. Gurvitz, Phys. Rev. B 56, 15215 (1997); S. Pascazio and P. Facchi, Acta Phys. Slov. 49, 557 (1999); M. Lewenstein and K. Rzążewski, Phys. Rev. A 61, 022105 (2000); O.V. Prezhdo, Phys. Rev. Lett. 85, 4413 (2000); P. Facchi, H. Nakazato, and S. Pascazio, ibid. 86, 2699 (2001); I. Antoniou, E. Karpov, G. Pronko, and E. Yarevsky, Phys. Rev. A 63, 062110 (2001).

[3] A. Luis and L.L. Sánchez-Soto, Phys. Rev. A 57, 781 (1998).

[4] A.G. Kofman and G. Kurizki, Acta Phys. Slov. 49, 541 (1999); A.D. Panov, Phys. Lett. A 260, 441 (1999); J. Ruseckas and B. Kaulakys, Phys. Rev. A 63, 062103 (2001); P. Facchi and S. Pascazio, e-print quant-ph/0101044.

[5] A.G. Kofman and G. Kurizki, Nature (London) 405, 546
(2000).

[6] A. Luis, Phys. Rev. A 64, 032104 (2001).

[7] S. Slijkhuis, G. Nienhuis, and R. Morgenstern, Phys. Rev. A 33, 3977 (1986); W.M. Itano, D.J. Heinzen, J.J. Bollinger, and D.J. Wineland, ibid. 41, 2295 (1990); B. Nagels, L.J.F. Hermans, and P.L. Chapovsky, Phys. Rev. Lett. 79, 3097 (1997); K. Mølhave and M. Drewsen, Phys. Lett. A 268, 45 (2000); T. Nakanishi, K. Yamane, and M. Kitano, e-print quant-ph/0103034; P. Valente, H. Failache, and A. Lezama, e-print quant-ph/0107007.

[8] S.R. Wilkinson, C.F. Bharucha, M.C. Fischer, K.W. Madison, P.R. Morrow, Q. Niu, B. Sundaram, and M.G. Raizen, Nature (London) 387, 575 (1997); M.C. Fischer, B. Gutiérrez-Medina, and M.G. Raizen, e-print quant-ph/0104035.

[9] C.K. Hong and L. Mandel, Phys. Rev. A 31, 2409 (1985); J. 
Peřina, Quantum Statistics of Linear and Nonlinear Optical Phenomena (Kluwer Academic, Dordrecht, 1991); L. Mandel and E. Wolf, Optical Coherence and Quantum Optics (Cambridge University Press, Cambridge, England, 1995).

[10] J. Peřina, Z. Hradil, and B. Jurčo, Quantum Optics and Fundamentals of Physics (Kluwer Academic, Dordrecht, 1994); V. Peřinová, A. Lukś, and J. Peřina, Phase in Optics (World Scientific, Singapore, 1998).

[11] A. Luis and J. Peřina, Phys. Rev. Lett. 76, 4340 (1996).

[12] J. Peřina and J. Křepelka, J. Mod. Opt. 39, 2405 (1992); J. Peřina, Jr. and J. Peřina, in Progress in Optics, edited by E. Wolf (North-Holland, Amsterdam, 2000), Vol. 41, p. 361.
[13] A. Yariv, Quantum Electronics (Wiley, New York, 1989).

[14] S.B. Cavalcanti, G.A. Barbosa, and J.M. Hickmann, Proc. SPIE 3749, 796 (1999); S. B. Cavalcanti, J. M. Hickmann, and G. A. Barbosa (private communication).

[15] J.F. Poyatos, J.I. Cirac, and P. Zoller, Phys. Rev. Lett. 77, 4728 (1996); M.B. Plenio, P.L. Knight, and R.C. Thompson, Opt. Commun. 123, 278 (1996); C.J. Myatt, B.E. King, Q.A. Turchette, C.A. Sackett, D. Kielpinski, W.M. Itano, C. Monroe, and D.J. Wineland, Nature (London) 403, 269 (2000); Q.A. Turchette, C.J. Myatt, B.E. King, C.A. Sackett, D. Kielpinski, W.M. Itano, C. Monroe, and D.J. Wineland, Phys. Rev. A 62, 053807 (2000). 